\title{
Modeling and Simulation of Non-Contact Base Excited AFM
}

\author{
Mohammad Reza Bahrami* \\ Innopolis University, Innopolis 420500, Russia
}

\begin{abstract}
AFM has some unique properties such as higher spatial resolution, mapping even a single molecule, simple sample preparation, scanning in different types of medium, and can obtain a 3D scan of the sample surface. Therefore, with the help of AFM, one can obtain a unique understanding of the structure and functional behavior of materials. In this article, to construct the mathematical model of the base excited AFM cantilever mass spring system (lumped parameter model) is used and the solution obtained by the method of Multiple scales. Here, in this work, we consider the AFM operates in the non-contact mode. To study the effect of the non linearity, amplitude of excitation, and damping coefficient, frequency response equation obtained.
\end{abstract}

\section{Introduction}

Atomic force microscope (AFM) which is a nanoscale prober was invented in 1986 [1] and serves to investigate nanoscale surfaces. AFM in contrast with other types of microscopes utilizes a sharp nanoscale tip instead of a light or electron beam. AFM can discover/find changes at a spatial resolution of up to a million X because of the sharp nanoscale tip. In comparison with other types of conventional microscopes has some unique properties such as higher spatial resolution, mapping even a single molecule, simple sample preparation, scanning in different types of medium (for example in liquids). And last not least, by AFM one can obtain a 3D scan of the sample surface. Therefore, with the help of AFM, one can obtain a unique understanding of the structure and functional behavior of materials [2, 3].

AFM is also known as a spacious technique that can be utilized to study the mechanical features of the specimen. These characteristics of AFM make it unique and a powerful tool that can be used in various broad types of science and technology like material science, microbiology, nanofabrication, etc., for a wide range of purposes [4-6].

The main idea of AFM is to utilize a sharp nanoscale tip at the end of the flexible micro cantilever moving near or in contact with the surface of the specimen. The specimen is located on the mechanical scanner, usually a piezoelectric, which provides accurate move in three dimensions. While the tip is moving on the sample surface, the interaction force between the tip and the sample is sensed by the tip and caused deflection on the cantilever beam. To detect these deflections, a laser beam is emitted from a laser diode into the back of

* Corresponding author: mo.bahrami@innopolis.ru 
the cantilever, and the reflected laser beam goes to the photodiode to sense the angular deflection of the cantilever. The cantilever deflection magnitude varies according to the changes of the position of the tip to the surface of the specimen, i.e. the interaction force between the tip and the sample surface. Sensing these changes, AFM can construct the topography of the surface, in three dimensions. To keep the tip at a constant distance from the surface of the sample, a feedback control unit is utilized. The schematic of a conventional AFM is shown in Fig. 1.

Depending on the usage of the AFM, it can be operated in a variety of modes. In general, there exists two possible imaging modes; contact mode (static mode) and dynamic modes including tapping and non-contact modes.

In the non-contact mode, a stiff cantilever is vibrated while the tip is quite close to the sample but not touching it, i.e. in an attractive regime. To construct the topography of the sample, the system measures the resonant frequency or amplitude of the cantilever difference. The cantilever is vibrated at a frequency close to the resonance frequency while the amplitude of oscillation is typically a few nanometers. In this work, we use the van der Waals force, which is strongest when the tip is close to the surface of the sample acts to reduce the resonance frequency or amplitude of the cantilever. The idea is to keep tip to sample distance constant. This can be accomplished by using this reduction in the resonance frequency and utilizing the feedback loop (control) system to keep the oscillation amplitude or frequency constant as well. After that, by measuring the tip-to-sample distance at each data point we can make a topographic scan of the sample surface [7, 8].

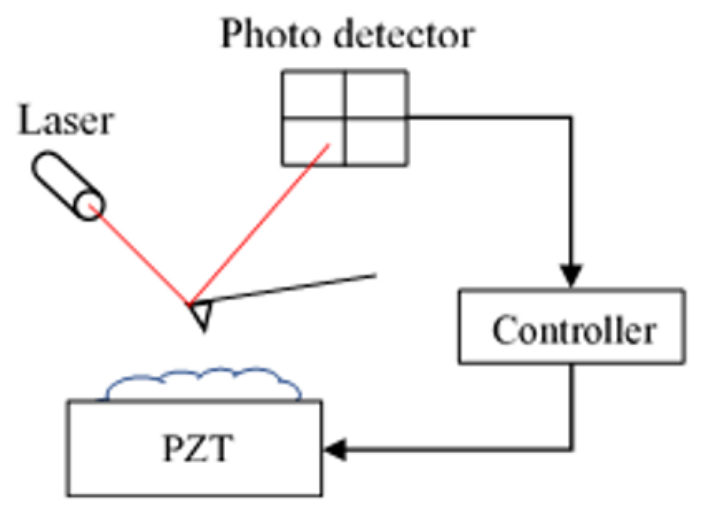

Fig. 1. Schematic of a typical AFM.

AFM micro-cantilever is one of the most important members of a conventional AFM that has a high impact on the operation of AFM. Therefore, to have an AFM with effective performance, it is needed to conduct a full dynamic analysis of the cantilever.

Different methods have been used to investigate the dynamic behavior of the cantilever in the different modes of operation [9-13]. In this article, to construct the mathematical model of the base excited AFM cantilever lumped parameter model is used and solved by the method of Multiple scales. Here, in this work, we consider the AFM operates in the noncontact mode. To study the effect of the non linearity, amplitude of excitation, and damping coefficient, frequency response equation obtained.

\section{Modeling of Non Contact Base Excited AFM Cantilever}

To model the AFM cantilever and find the equation of motion for the non contact base excited AFM Cantilever generally one can use two simplifications. In the first model, one assumes that the cantilever deflection is caused by a static point load, applying at the free end of the 
cantilever. The one degree of freedom mass spring model is derived considering the corresponding static stiffness. To construct the second model, one may discretize the classical beam equation according to the eigenmodes of the cantilever beam and derive the multiple degrees of freedom models. Each of these models has its pros and cons [14, 15] that are beyond the goal of this work. In work [16] presented how to determine the unique equivalent masses and stiffnesses using the virtual work for the mass spring model (in this article we use the same model as shown in Fig. 2). The resulting equation of motion for this system may be written in the following form [17 - 20]:

$$
m \frac{\mathrm{d}^{2} z}{\mathrm{~d} \tau^{2}}+b \frac{\mathrm{d}}{\mathrm{d} \tau}(z-P)+k(z-P)=f_{\text {tip }}(z(\tau))
$$

where $m$ is the equivalent mass, $k$ is the equivalent stiffnesses and $b$ is the equivalent corresponding damping coefficient of the micro-cantilever. It should be mentioned that the external harmonic force, to excite the base, is presented by $P$ and the interaction force between the cantilever tip and the surface of the sample is presented $f_{\text {tip }}$ in Eq.1. We use van der Waals force as the interaction force. These forces are in the following format:

$$
\begin{aligned}
& P(\tau)=p \cos \omega \tau, \\
& f_{\text {tip }}(z(\tau))=\frac{A_{H} R}{6\left(z_{0}-z\right)^{2}} . \\
& \hline \sum_{\text {Sample Surface }}
\end{aligned}
$$

Fig. 2. Lumped-parameters model of the cantilever AFM system.

Now by substituting Eq.2 in Eq.1 we have the full equation of the motion as:

$$
m \frac{d^{2} z}{d \tau^{2}}+b \frac{d z}{d \tau}+k z=\frac{A_{H} R}{6\left(z_{0}-z\right)^{2}}+b p \omega \sin \omega \tau-k p \cos \omega \tau .
$$

One can determine the equilibrium point of the static system by putting time terms and $p$, excitation amplitude, in Eq.3 to zero. We get: 


$$
\begin{aligned}
& Z_{s}=\frac{3}{2}(2 H)^{\frac{1}{3}}, \\
& H=\frac{A_{H} R}{6 k} .
\end{aligned}
$$

To find the dimensionless equation to avoid the influence of other parameters such as temperature, we use the following dimensionless variables:

$$
\begin{aligned}
& x=\frac{Z}{Z_{s}}, \\
& t=\omega_{0} \tau, \\
& F=\frac{p}{Z_{s}}, \\
& \zeta=\frac{z_{0}}{Z_{s}}, \\
& \omega_{0}=\sqrt{\frac{k}{m}}, \\
& \phi=\frac{\omega}{\omega_{0}}, \\
& B=\frac{b}{m \omega_{0}},
\end{aligned}
$$

and substitute them, Eq.5, in Eq.3 to get the dimensionless equation of system motion as:

$$
\ddot{x}+B \dot{x}+x=\frac{c}{(\zeta-x)^{2}}+F B \phi \sin \phi t-F \cos \phi t .
$$

It should be noted that in Eq.6 dots are derivative with respect to the new dimensionless variable $t$ and $c$ is a constant coefficient $(c=4 / 27)$.

\section{Method of Multiple Scales}

In this paper, to solve Eq.6 we use the method of multiple scales. This method is used to make valid approximations to disturbance problems that their solutions are influenced by widely different scales. To accomplish this, in this method, we introduce fast and slow variables and treat them as they are independents [21, 22].

To use the method of multiple scales, it is needed to scale the Eq.6 as:

$$
\ddot{x}+x=\epsilon\left(\frac{c}{(\zeta-x)^{2}}-B \dot{x}\right)+F B \phi \sin \phi t-F \cos \phi t .
$$

Hereafter we use the detuning parameter $\sigma$ instead of using excitation frequency. The detuning parameter $\sigma$ is a parameter that describes the nearness of $\phi$ to $\omega_{0}$. Here, we have: 


$$
\phi=1+\epsilon \sigma
$$

The time scales are introduced as:

$$
T_{n}=\epsilon^{n} t \quad \text { for } n=0,1, \ldots
$$

Now, we conduct derivatives with respect to $t$ expressed in terms of the partial derivatives with respect to $T_{n}$, i.e.

$$
\begin{aligned}
& \frac{d}{d t}=D_{0}+\epsilon D_{1}+2 \epsilon D_{2}+\ldots, \\
& \frac{d^{2}}{d t^{2}}=D_{0}^{2}+2 \epsilon D_{0} D_{1}+\epsilon^{2}\left(D_{1}+2 D_{0} D_{1}\right)+\ldots
\end{aligned}
$$

One may write the approximate solution of Eq.7 in the following format:

$$
x(t ; \epsilon)=x_{0}\left(T_{0}, T_{1}\right)+\epsilon x_{1}\left(T_{0}, T_{1}\right) .
$$

Knowing the fact that $T_{0}=t$ and $T_{1}=\epsilon t$, one may write the second and third term of the right-hand side in terms of $T_{0}$ and $T_{1}$ as:

$$
E\left(T_{0}, T_{1}\right)=F B(1+\epsilon \sigma) \sin \left(T_{0}+\sigma T_{1}\right)-F \cos \left(T_{0}+\sigma T_{1}\right) .
$$

We use the Taylor series expansion to approximate the first term on the right-hand side of Eq.7.

Integrating Eq.11 and Eq.12 into Eq.7 and equating the coefficients of $\epsilon^{0}$ and $\epsilon^{1}$ on both sides, we find

$$
\begin{gathered}
D_{0}^{2} x_{0}+x_{0}=0, \\
D_{0}^{2} x_{1}+2 D_{0} D_{1} x_{0}+x_{1}=\frac{c}{\zeta}+2 \frac{c}{\zeta^{2}} x_{0}+3 \frac{c}{\zeta^{3}} x_{0}^{2}+4 \frac{c}{\zeta^{4}} x_{0}^{3}-\ldots \\
\ldots-B D_{0} x_{0}+F B \sin \left(T_{0}+\sigma T_{1}\right)-F \cos \left(T_{0}+\sigma T_{1}\right) .
\end{gathered}
$$

The solution of Eq.13 may be expressed as:

$$
x_{0}=A\left(T_{1}\right) \exp \left(i T_{0}\right)+\bar{A}\left(T_{1}\right) \exp \left(-i T_{0}\right)
$$

and insert it into Eq.14, we have:

$$
\begin{aligned}
& D_{0}^{2} x_{1}+x_{1}= \\
& 3 \frac{c}{\zeta^{3}}\left\{A\left(T_{1}\right)^{2} \exp \left(2 i T_{0}\right)+2 A\left(T_{1}\right) \bar{A}\left(T_{1}\right)\right\}+12 \frac{c}{\zeta^{4}} A\left(T_{1}\right)^{3} \exp \left(3 i T_{0}\right) \\
& +\exp \left(i T_{0}\right)\left\{-\frac{1}{2} F \exp \left(\sigma T_{1}\right)-\frac{i F B}{2} \exp \left(\sigma T_{1}\right)-i B A\left(T_{1}\right)+\right. \\
& \left.12 \frac{d}{\zeta^{4}} A\left(T_{1}\right)^{2} \bar{A}\left(T_{1}\right)+2 \frac{c}{\zeta^{2}} A\left(T_{1}\right)-2 i A^{\prime}\left(T_{1}\right)\right\}+\frac{c}{\zeta}+c c .
\end{aligned}
$$
terms.

It should be noted that in Eq.16, $c c$, is designated to the complex conjugate of preceding 
Now, it is needed to get rid of secular terms from the particular solution of Eq.16. To accomplish this, we set:

$$
\begin{aligned}
& -2 i A^{\prime}\left(T_{1}\right)+2 \frac{c}{\zeta^{2}} A\left(T_{1}\right)+12 \frac{c}{\zeta^{4}} A\left(T_{1}\right)^{2} \bar{A}\left(T_{1}\right) \\
& -i B A\left(T_{1}\right)-\frac{F}{2}(B i-1) \exp \left(i \sigma T_{1}\right)=0
\end{aligned}
$$

Introducing $A\left(T_{1}\right)=\frac{1}{2} a \exp (i \beta)$, inserting into Eq.17, and separating the real and imaginary parts, one obtains

$$
\begin{gathered}
a^{\prime} \cos \beta-a \beta^{\prime} \sin \beta-\frac{3}{2} \frac{c}{\zeta^{4}} a^{3} \sin \beta+\frac{1}{2} B a \cos \beta \\
-\frac{c}{\zeta^{2}} a \sin \beta+\frac{F}{2}\left(\cos \sigma T_{1}+B \sin \sigma T_{1}\right)=0 \\
a^{\prime} \sin \beta+a \beta^{\prime} \cos \beta+\frac{3}{2} \frac{c}{\zeta^{4}} a^{3} \cos \beta+\frac{1}{2} B a \sin \beta \\
+\frac{c}{\zeta^{2}} a \cos \beta+\frac{F}{2}\left(\sin \sigma T_{1}-B \cos \sigma T_{1}\right)=0
\end{gathered}
$$

where $a$ and $\beta$ are real variables.

We solve Eq.18 and Eq.19 for $a^{\prime}$ and $\beta^{\prime}$. We obtain:

$$
\begin{aligned}
& a^{\prime}=0.5 F\left\{B \sin \left(\beta-\sigma T_{1}\right)-\cos \left(\beta-\sigma T_{1}\right)\right\}-0.5 B a, \\
& \beta^{\prime}=\frac{F}{2 a}\left\{B \cos \left(\beta-\sigma T_{1}\right)+\sin \left(\beta-\sigma T_{1}\right)\right\}-\frac{c}{\zeta^{2}}-\frac{3}{2 \zeta^{4}} a^{2} c
\end{aligned}
$$

Here, by introducing a new variable $\beta-\sigma T_{1}=\gamma$, we can rewrite Eq.20 and Eq.21 in the following forms:

$$
\begin{gathered}
a^{\prime}=0.5 F\{K \cos (\gamma+\theta)\}-0.5 B a, \\
\beta^{\prime}=\frac{F}{2 a}\{K \sin (\gamma+\theta)\}-\frac{c}{\zeta^{2}}-\frac{3}{2 \zeta^{4}} a^{2} c-\delta, \\
K=\sqrt{1+B^{2}}, \\
\theta=\arctan (-B) .
\end{gathered}
$$

Introducing $\gamma+\theta=\Omega$ and setting $a^{\prime}=\beta^{\prime}=0$ we can find the steady-state motions as:

$$
\begin{aligned}
& B a=F K \cos \Omega, \\
& \left(\frac{c}{\zeta^{2}}+\frac{3}{2 \zeta^{4}} a^{2} c+\delta\right) 2 a=-F K \sin \Omega
\end{aligned}
$$


squaring and summing Eq.25 we obtain the implicit equation of amplitude as the function of frequency:

$$
[B a]^{2}+\left[\left(\frac{c}{\zeta^{2}}+\frac{3}{2 \zeta^{4}} a^{2} c+\delta\right) 2 a\right]^{2}=(F K)^{2} .
$$

Now, we use Eq. 26 to investigate the effect of the nonlinearity, $\zeta$, the amplitude of excitation, $F$ and damping coefficient, $b$, on the behavior of the cantilever under the base excitation.

Fig.3a presents the comparison of the linear case with the nonlinear case. By set the nonlinear variable to zero we reach the linear case as shown.

In Fig.3b, the effect of excitation force, $F$ has been shown. As we expect, by increasing the magnitude of the external force, the amplitude of the response increases as well.

In contrast with the external force, by increasing the corresponding damping coefficient, the amplitude of response decreases as we expected (Fig.3c).

In nonlinear systems with harmonic excitation may happen a phenomenon in where the magnitude of the response motion can jump upward or downward as the input variable, here the detuning parameter, is varying. The graph of the amplitude of response versus the detuning parameter in our case follows a hysteresis loop as shown in Fig.3d by arrows and numbers [23, 24].

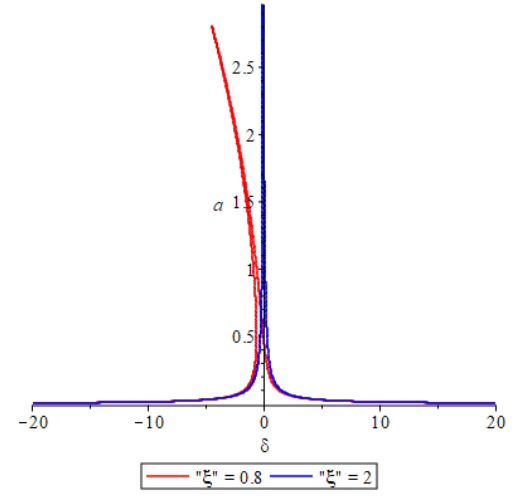

(a)

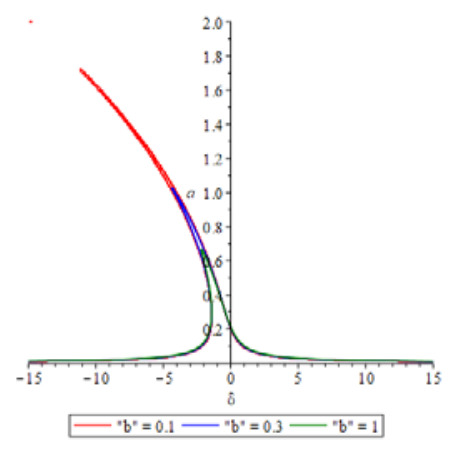

(c)

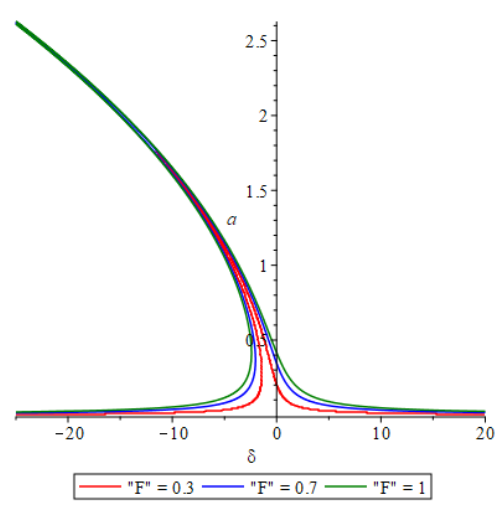

(b)

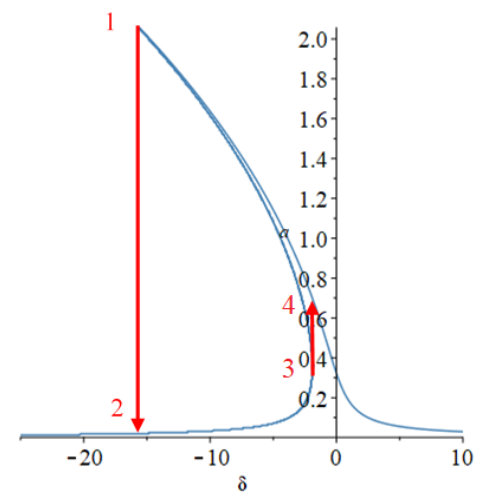

(d)

Fig. 3. (a) Effect of non linearity, (b) effect of excitation, (c) effect of damping (d) jumping phenomenon. 


\section{Conclusion}

Atomic force microscope (AFM) is a nanoscale prober that serves to study nanoscale surfaces. AFM utilizes a sharp nanoscale tip instead of a light or electron beam to discover/find changes at a spatial resolution of up to a million $\mathrm{X}$ because of the sharp nanoscale tip. This work aims to construct the mathematical model of the base excited AFM cantilever to study the effect of nonlinearity, damping coefficient, and external force on the response of the system. To accomplish this, we use lumped model to find the equation of motion of the system. Then we utilized the multiple scales method to obtain the frequency response equation.

\section{References}

1. G. Binnig, C. F. Quate, and C. Gerber, Atomic force microscope, Physical review letters, 56 (9) (1986) p. 930.

2. S. Liu and Y. Wang, Application of AFM in microbiology: a review, Scanning, 32 (2) (2010) pp. 61-73.

3. Y. Seo and W. Jhe, Atomic force microscopy and spectroscopy, Reports on Progress in Physics, 71 (1) (2007) p. 016101.

4. J. L. Hutter and J. Bechhoefer, Calibration of atomic force microscope tips, Review of Scientific Instruments, 64 (7) (1993) pp. 1868-1873.

5. B. Drake, C. Prater, A. Weisenhorn, S. Gould, T. Albrecht, C. Quate, D. Cannell, H. Hansma and P. Hansma, Imaging crystals, polymers, and processes in water with the atomic force microscope, Science, 243 (4898) (1989) pp. 1586-1589.

6. M. Marrese, V. Guarino and L. Ambrosio, Atomic force microscopy: a powerful tool to address scaffold design in tissue engineering, Journal of functional biomaterials, 8 (1) (2017) p. 7.

7. N. Jalili and K. Laxminarayana, A review of atomic force microscopy imaging systems: application to molecular metrology and biological sciences, Mechatronics, 14 (8) (2004) pp. 907-945.

8. H. G. Hansma and L. Pietrasanta, Atomic force microscopy and other scanning probe microscopies, Current opinion in chemical biology, 2 (5) (1998) pp. 579-584.

9. M. R. Bahrami and A. B. Abeygunawardana, Modeling and simulation of dynamic contact atomic force microscope, in Advances in Mechanical Engineering, Springer, pp. 109-118 (2019).

10. M. R. Bahrami and B. Abeygunawardana, Modeling and simulation of tapping mode atomic force microscope through a bond graph, in Advances in Mechanical Engineering, Springer, pp. 9-15 (2018).

11. M. R. Bahrami, A. Ramezani and K. G. Osquie, Modeling and simulation of noncontact atomic force microscope, in ASME 2010 10th Biennial Conference on Engineering Systems Design and Analysis, American Society of Mechanical Engineers Digital Collection (2010), pp. 565-569.

12. A. Sebastian, M. Salapaka, D. Chen and J. Cleveland, Harmonic analysis based modeling of tapping mode AFM, in Proceedings of the 1999 American Control Conference (Cat. No. 99CH36251), IEEE (1999), vol. 1, pp. 232-236.

13. S. Belikov and S. Magonov, Classification of dynamic atomic force microscopy control modes based on asymptotic nonlinear mechanics, in 2009 American Control Conference, IEEE (2009), pp. 979-984. 
14. S. Hu and A. Raman, Analytical formulas and scaling laws for peak interaction forces in dynamic atomic force microscopy, Applied Physics Letters, 91 (12) (2007) p. 123106.

15. F. J. Gießibl, A direct method to calculate tip-sample forces from frequency shifts in frequency modulation atomic force microscopy, Applied Physics Letters, 78 (1) (2001) pp. 123-125.

16. J. Melcher, S. Hu and A. Raman, Equivalent point mass models of continuous atomic force microscope probes, Applied Physics Letters, 91 (5) (2007) p. 053101.

17. M. R. Bahrami, Dynamic analysis of atomic force microscope in tapping mode, Vibroengineering PROCEDIA, 32 (2020) pp. 13-19.

18. M. R. Bahrami, Modeling of noncontact atomic force microscope with two term excitations, in IOP Conference Series: Materials Science and Engineering, IOP Publishing (2020), vol. 971, p. 042052.

19. M. R. Bahrami, Nonlinear dynamic analysis of noncontact atomic force microscope, in 2020 4th Scientific School on Dynamics of Complex Networks and their Application in Intellectual Robotics (DCNAIR), IEEE (2020), pp. 49-52.

20. M. R. Bahrami, Modeling of physical systems through bond graphs, st, Petersburg: Polytech Uni Pub, St. Petersburg.

21. C. M. Bender and S. A. Orszag, Advanced mathematical methods for scientists and engineers I: Asymptotic methods and perturbation theory, Springer Science \& Business Media (2013).

22. A. H. Nayfeh, Perturbation Methods, John Wiley \& Sons (2008).

23. M. Markakis, The jump phenomenon associated with the dynamics of the duffing equation, Physics Open, 5 (2020) p. 100042.

24. A. H. Nayfeh and D. T. Mook, Nonlinear oscillations, John Wiley \& Sons (2008). 